\title{
Detection of Semen (Human and Boar) and Saliva on Fabrics by a Very High Powered UV-/VIS-Light Source
}

\author{
Anja Fiedler*,1, Jessica Rehdorf ${ }^{2}$, Florian Hilbers ${ }^{3}$, Lena Johrdan ${ }^{4}$, Carola Stribl ${ }^{5}$ and \\ Mark Benecke*,
}

\author{
${ }^{1}$ Paul Flechsig Institute, University of Leipzig, Germany \\ ${ }^{2}$ Institute for Chemistry and Biochemistry, University of Greifswald, Germany \\ ${ }^{3}$ University of Osnabrück, Germany \\ ${ }^{4}$ University of Bochum, Germany \\ ${ }^{5}$ University of Regensburg, Germany \\ ${ }^{6}$ Internat. Forensic Research \& Consulting, Postfach 250411, 50520 Cologne, Cologne, Germany
}

\begin{abstract}
A possible method to detect body fluids in forensic cases of sexual assault or abuse is by using forensic light sources, which emit certain wavelengths and excite body fluids to fluoresce. However, the success of the visualization of the fluorescence signal can be significantly reduced when unfavorable conditions - especially daylight - are present or used.

We studied the detection of fresh semen (human, boar) and saliva (human) on different and colored types of fabric. The stained samples were stored for 3 and 5 weeks, respectively, and some were additionally washed with detergent at $30^{\circ} \mathrm{C}$ $\left(86^{\circ} \mathrm{F}\right)$. The portable forensic light source Lumatec Superlight 400 which emits wavelengths from $320 \mathrm{~nm}$ (UV) to $700 \mathrm{~nm}$ (visible light, VIS) and colored goggles and filters were used.

The very high intensity light source detected semen and saliva in darkness and daylight. No difference were found in samples stored 3 and 5 weeks, respectively. Best results for semen and saliva were obtained using wavelengths between 415-490 nm. For general search for body fluids, excitation of 350-500 $\mathrm{nm}$ is preferable. With appropriate goggles (orange $(>500 \mathrm{~nm})$ or red $(>590)$ ) semen could be detected in $100 \%$ and saliva in $60 \%$ of the cases. Washing the samples with detergent removed the biological stains in $75 \%$ of the cases. The darker the color, the more the fluorescence signal was absorbed. The type of the fabric had however no significant effect on detection of semen and saliva. The recognition rate of saliva was much better than reported for other light sources.
\end{abstract}

An unexpected observation on the side was that the fluorescence signal of boar semen was clearly weaker than that of human semen, although the amount of sperm cells per $\mathrm{ml}$ is 50-150 million for humans and 25-300 million for boar.

\section{INTRODUCTION}

Detection of semen and saliva in forensic cases (mostly sexual assault or abuse) is critical for evidence collection and possible DNA fingerprinting. Semen and saliva are known to fluoresce when exposed to light of certain wavelengths. Wood's lamp for example emits ultra violet (UV) light $(\sim 320-400 \mathrm{~nm})$ and was reported in 1919 to let semen fluoresce [1]. However, the effectiveness of Wood's lamp was challenged by several authors [2-4]. The excitation spectrum of semen is reported to be from 300 to $500 \mathrm{~nm}$ and the emission peaks of semen are reported to be around $460-520 \mathrm{~nm}$ [2-5] or 400-700 nm [6]. The emission spectra of saliva are documented to have a maximum at $345-355 \mathrm{~nm}$ when excited at $282 \mathrm{~nm}$ on skin [7]. The detection of saliva by detection of emitted light is more difficult. Auvdel [8] reports a success rate of $30 \%$ for an argon ion laser with 454.5-

*Address correspondence to these authors at the Paul Flechsig Institute, University of Leipzig, Germany; Tel: +49 178 5257255;

E-mail: afiedler@uni-leipzig.de

Internat Forensic Research \& Consulting, Cologne, Germany; Tel: +49 173 287 3136; E-mail: forensic@benecke.com
$514.5 \mathrm{~nm}$ excitation. Saliva can be detected by amylase tests as well but this may lead to consumption of the stain, especially in small stains; success rates of amylase detection depend very much on the system used; even though, it is sometimes used as a routine method in high throughput laboratories. A noninvasive detection using a light source is therefore not only helpful but also prevents loss of stains for DNA typing.

For our experiments we used a newly developed, very high intensity forensic light source (Lumatec Superlight 400) which emits light between $320 \mathrm{~nm}$ (UV) and $700 \mathrm{~nm}$ (visible light) using wavelengths filters. Because of the high intensity and the portability of the unit, it can be used on crime scenes even at daylight. We focused on the detection of semen and saliva on fabric because body fluids are mostly deposited on the cloth of the victim or/and on other possible fabrics left at crime scenes or recovered from offenders. We also chose body fluids on fabric because they are less likely to be removed compared to body fluids on skin which results in a longer time interval for detection, and for availability of the biological material. The type and the color of the fabric might influence the fluorescence signal $[6,9]$ and possibly 
prevent the detection of biological stains. Therefore, we tested the detection of semen and saliva on several fabrics in different colors and patterns. The fabric stained with biological fluids was stored for 3 and 5 weeks prior to analysis. Some samples were washed with different detergents to check for visible remains of the detergents in the fabric as well as to check for success rates of detection after washing the cloth.

\section{MATERIALS AND METHODS}

\section{Semen, Saliva and Cloth Fabrics}

Ten commercially available pieces of clothing of different fabric types were tested: $3 \times 100 \%$ cotton; $3 \times 100 \%$ polyamide; $1 \times 100 \%$ polyester; $1 \times 95 \%$ cotton $/ 5 \%$ spandex; $1 \times 73 \%$ cotton $/ 24 \%$ polyamide, $1 \times 3 \%$ spandex; $1 \times 80 \%$ polyamide $/ 20 \%$ spandex (Fig. 1). This resulted in different colors and color combinations of fabric, including white and black. The fabric was classified into three groups: bright (white, bright blue and pink), medium (blue, green, yellow, orange, red) and dark (black, brown).

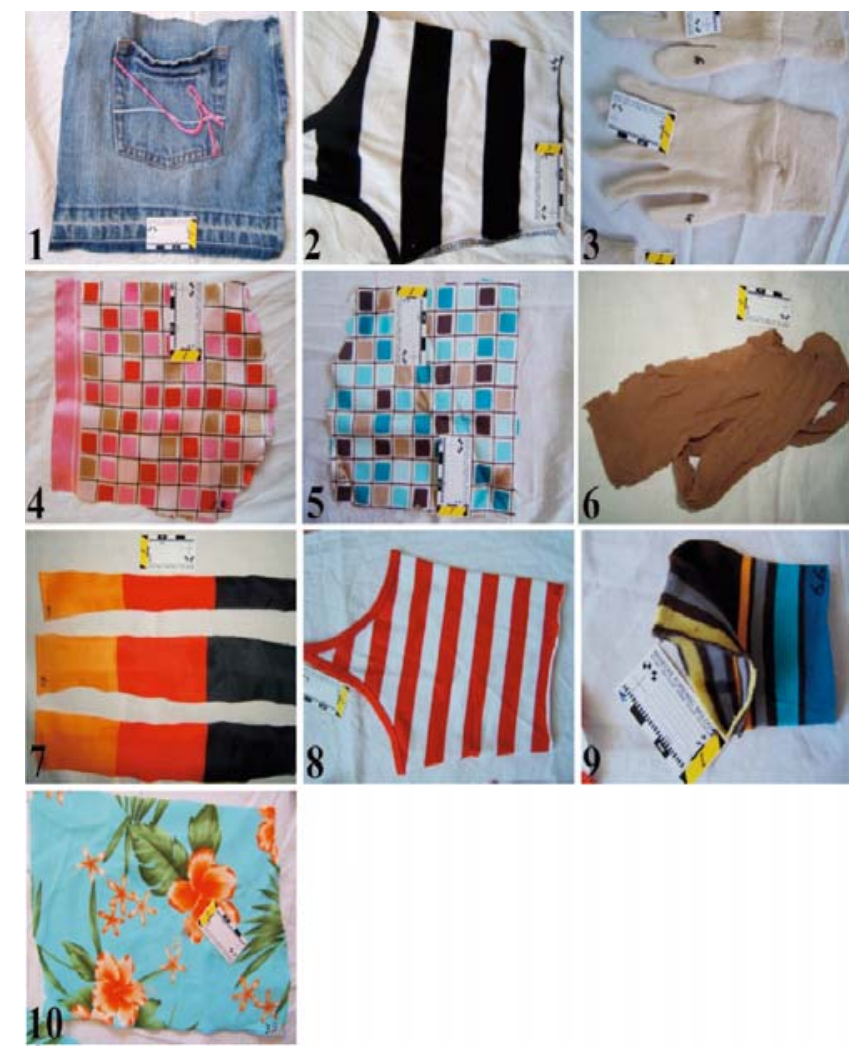

Fig. (1). Types of fabric used for semen and saliva detection. (1-3) $100 \%$ cotton; (4-6) $100 \%$ polyamide; (7) $100 \%$ polyester; (8) $95 \%$ cotton, $5 \%$ spandex; (9) $73 \%$ cotton, $24 \%$ polyamide, $3 \%$ spandex; (10) $80 \%$ polyamide, $20 \%$ spandex.

Human semen samples from a single donor and one boar (Sus scrofa domestica) were used. Ethical clearance was obtained by informed consent of the human donor. The semen samples were kept refrigerated at $+10^{\circ} \mathrm{C}$ (i.e., not frozen) and were applied $12 \mathrm{hrs}$ (human) and $20 \mathrm{hrs}$ (boar) after collection. Saliva from a single human donor was applied directly on the fabric. The amount of sperm and saliva was sufficient to be visible as long as it was wet (Fig. 3). The fresh stains were photographed on the fabric to compare the fluorescence signals of these locations before and after the forensic light analysis. Semen and saliva stains on the clothing were allowed to dry at $+25^{\circ} \mathrm{C}$ for six hours, then folded and stored at room temperature in paper bags in a dark room for 3 weeks and 5 weeks, respectively.

To test the influence of washing on the detection of the stains, a set of samples was washed at $30^{\circ} \mathrm{C}\left(86^{\circ} \mathrm{F}\right)$ in a commercial household washing machine after storage. The low temperature was chosen because it is a widely used presetting of washing machine programs in Europe. Normal "full" detergent (containing oxygen-based bleach) or mild detergent (free of bleach) was used. The cloths were dried for one day in a dimmed room (no tumbler). The experimental comparisons for each fabric type (cloths samples were cut into pieces) were: a. Stored for 3 and 5 weeks, respectively, b. washed or unwashed and c. washed with "full" detergent or soft detergent.

Finally, the samples were analysed by UV and visible fluorescence light using the UV/VIS excitation wavelengths of the forensic light source (see below).

\section{Light Source}

To test the excitation of the samples, we used a newly developed, very high intensity light source manufactured by Lumatec GmbH, Germany (Fig. 2). The portable system is suitable for heavy use at crime scenes because of its low weight (approximately $6 \mathrm{~kg}$ ), a unusually robust full metal shell, compactness and a battery that allows 60 minutes of operation away from an electrical outlet. A mercury lamp inside of the unit produces a high intensity of UV (320$400 \mathrm{~nm}$ ) and visible light (400-700 nm) which was developed to detect biological stains even in daylight. An applicator at the end of the flexible light tube results in a beam focused on a spot or spread to a fan-like triangle.

Wavelengths can be chosen by ten adjustable filter positions (400-700, 350-500, 400-500, 320-400, 415, 440, 460, $490,550,570)$. In combination with the ten excitation settings, white $(>400 \mathrm{~nm})$, orange $(>500 \mathrm{~nm})$ and red $(>590 \mathrm{~nm})$ goggles were used to block the excitation light and to visualize the fluorescence more precisely. This leads to 30 combinations of wavelengths and goggles. For the unwashed cloth, an available lorgnette with five high pass filters was additionally used.

\section{Experimental Combinations}

The specimens were illuminated in a distance of approximately 0.7 meter. All tests for biological stains were carried out under blind conditions with three independent persons in a dark room and in a room with mostly daylight and daylight-type artificial light, respectively. The examiners had also to categorize the fluorescence intensity as "visible", "hardly visible" or "non-visible signal".

\section{RESULTS}

Using appropriate goggles, semen was detected in $100 \%$ and saliva in $60 \%$ of the cases on unwashed fabric. Semen was visualized in $55 \%$ of the cases with red goggles, in $50 \%$ with the orange goggles and in $10 \%$ with white goggles. Saliva was visualized in $67 \%$ of the cases with the red goggles, in $50 \%$ with orange goggles and in $20 \%$ with white goggles. 
The fluorescence intensity of saliva was weaker than that of semen. Selection of specific wavelengths (narrow bands) led to more precise emission spectra and an easier detection. The best results for detection of semen and saliva were obtained at an excitation band between 415-490 nm (Table 1). In this frequency band, the detection rate on unwashed clothing with red or orange goggles was about $65 \%$ for semen and $80 \%$ for saliva. For general search for body fluids, a broader excitation range with wavelengths of $350-500 \mathrm{~nm}$ led to better results. The fluorescence signal intensity was similar in samples that were stored for 3 compared to 5 weeks.

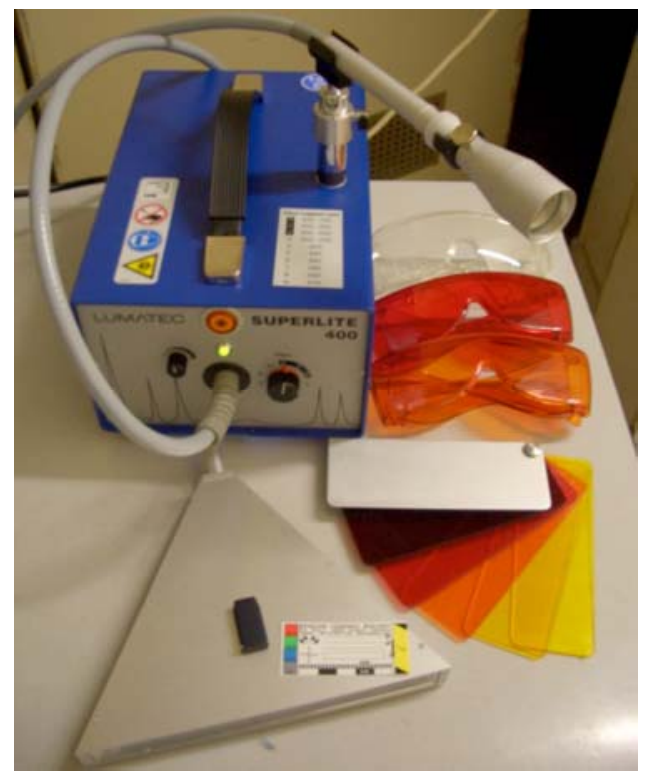

Fig. (2). Lumatec Superlite 400 forensic light source with spot or fan light guide applicator, tree high pass goggles and lorgnette with 5 filters.

After washing the cloth for 30 minutes at $30^{\circ} \mathrm{C}\left(86^{\circ} \mathrm{F}\right)$ with washing detergents, the detectable fluorescence signal of the biological stains was effectively reduced (normal "full wash" detergent: $85 \%$ reduction, mild detergent: $90 \%$ reduction). Due to the similar signal reduction of both detergents, the oxygen-based bleach contained in the normal "full wash" detergent seems not be of prime importance for the signal reduction.

Generally, the fluorescence signal was nearly as strong at daylight as in darkness in most cases. This means that we could reproduce the manufactur's claim that the intensity of the lamp allows the use of the Superlight 400 at crime scenes even at daylight. However filters that block the bright incident light (orange and red goggles) must be used to allow visualization of the fluorescence.

There was no significant difference in the detection rates of biological stains between the different types of fabrics. However, the color of the fabric did have an influence on the visualization of the stains. The darker the color, the lower the chance to detect biological traces. Especially pure black effectively absorbed excited and emitted fluorescence light so that both semen and saliva became nearly invisible on such cloth (Fig. 3). This was in accordance with the observations of Kobus et al. [9]. Bright and medium colored fabric showed good detection results for semen and saliva (see also [6]).

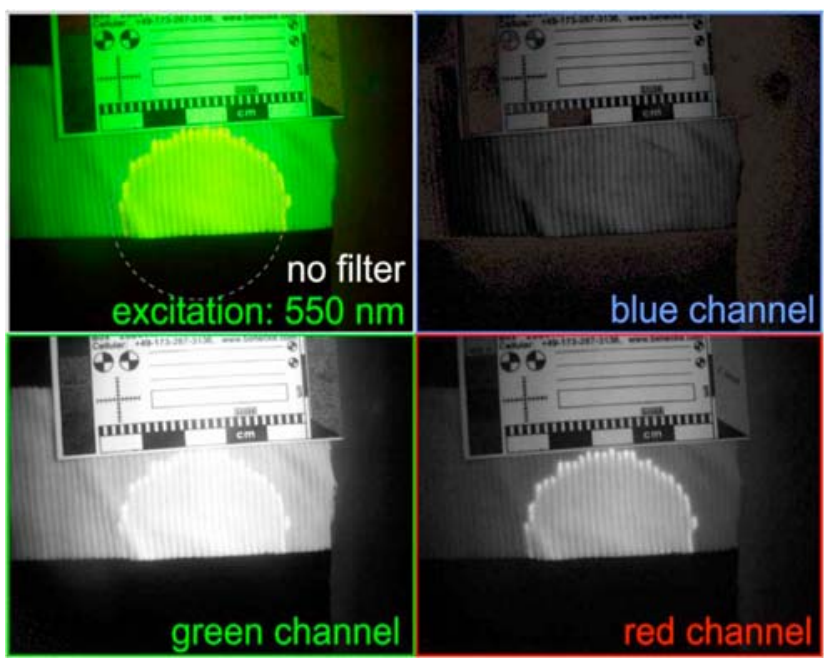

Fig. (3). Spot of human semen on a white and black striped cotton top excited with a wavelength of $550 \mathrm{~nm}$, no filter. The stain is clearly visible on the white stripe, but not on the black stripe. The splitting of the image into blue, green and red channels shows that the emission occurs from green to red light.

An unexpected observation on the side was that the fluorescence signal of boar semen was clearly weaker than that of human semen, although the amount of sperm cells per $\mathrm{ml}$ is 50-150 million for human and 25-300 million for boar, respectively [10].

\section{DISCUSSION}

Due to its unusually high intensity, the portable forensic light source Lumatec Superlight 400 detects biological stains in darkness and daylight and can therefore not only be used in the laboratory but also at crime scenes that cannot be darkened. Visualization is however only optimal when filters are chosen that block the bright excitation as well as the ambient light and allow only transmission of the wavelengths emitted and amplified from the stain. For semen and saliva, the orange bright pass goggles $(>500 \mathrm{~nm})$ work best. The red bright pass goggles $(>590 \mathrm{~nm})$ are better for visualization when both body fluids are present because they reduce the background light emitted from the cloth. Transparent white goggles do not aid in the detection of semen and saliva. With appropriate goggles, the Lumatec Superlight is as a very reliable test for the detection of semen on unwashed fabric. The fluorescence signal of saliva was generally weaker. However, the recognition rate of saliva was much better than reported for other light sources [8]. This might result from precise excitation and filtering, but is also due to the high intensity of the lamp. The use of this forensic light source is therefore a good nondestructive, presumptive test for saliva stains on unwashed cloth.

Washing the stained fabric even at low temperatures did often remove the biological stains. The tested types of fabric (cotton, polyester, polyamide, spandex) did not have remarkable influence on the fluorescence signal. Dark fabric colors however absorbed the light and reduced the chance of detection of the biological traces very much. Especially on pure 
Table 1. Recognition Rate with Different Goggles for Unwashed Fabric at Different Excitation Wavelengths

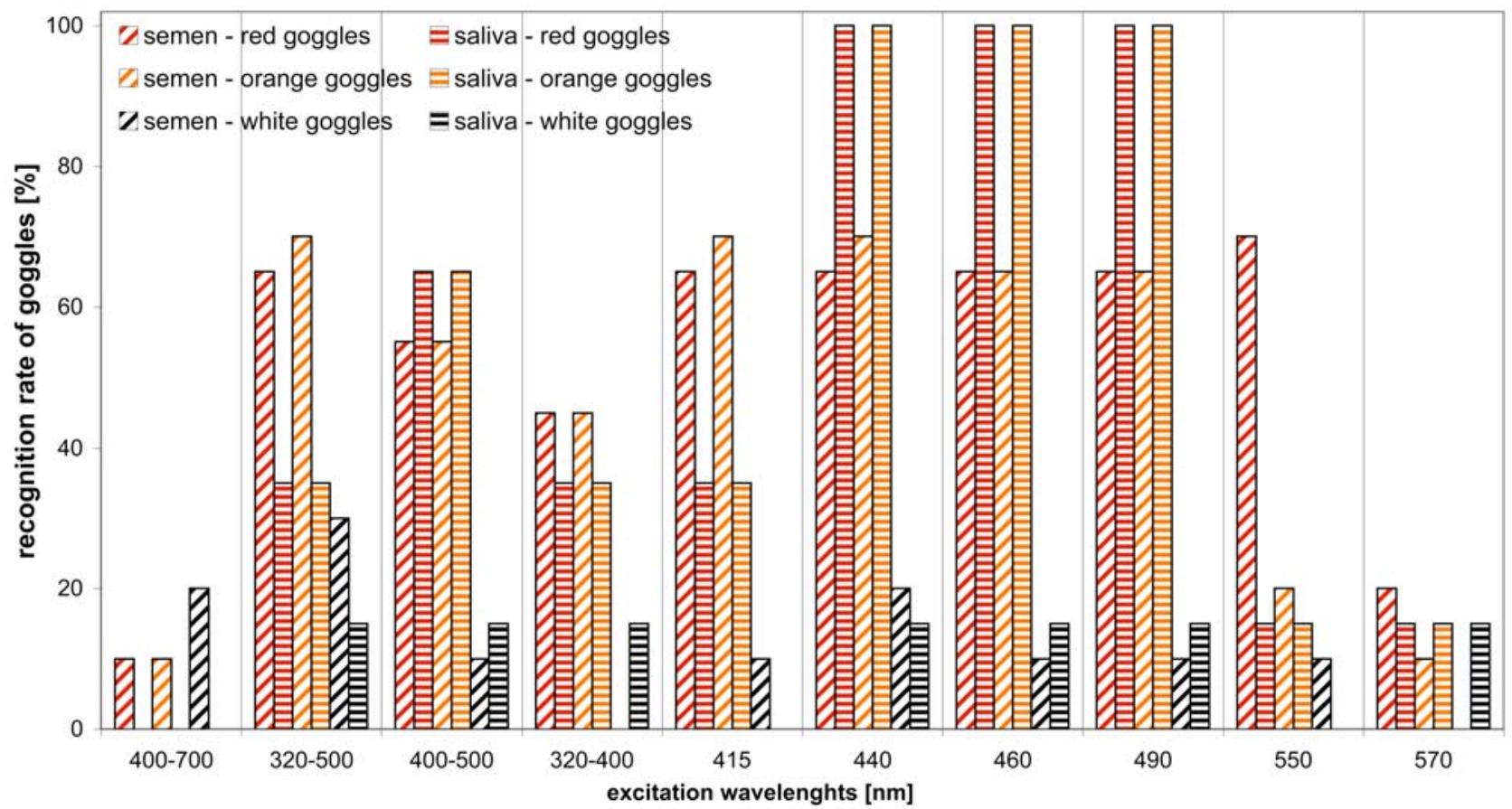

black fabric, the stains were not visible with any of the 10 possible excitations between 320 and $700 \mathrm{~nm}$.

Even though the amount of sperm cells of human and boar sperm are comparable, human semen had a brighter fluorescence. It seems that other components of the ejaculate (not the actual sperm cells) contribute to the visible emission of light. A reason might be the secretion of the bleb glands (fructose, phosphorylcholin, ergothionin, ascorbic acid, prostaglandin, proteins), the prostate gland (spermin, acid phosphatase, citric acid, cholesterol, phospholipids, fibrinolysis, glutamin acid, zinc) and of the epididymal area (carnitin, lecithin) [11]. For saliva secretion, proteins of the salivary glands $(\alpha$-amylase, lysozyme, lactoferrine, lactoperoxidasis, cystatins, histatins, mucins, lipase, proteinase, DNAse, RNases), electrolytes and other components like bacteria, epithelial cells, erythrocytes and leukocytes as well as food debris could contribute to the fluorescence [12].

\section{ACKNOWLEDGEMENT}

We wish to thank the anonymous referee for his valuable input.

\section{REFERENCES}

[1] Woods, R.W. J. de Physique Theor. et Appl., 1919, 59, 77-90.

[2] Stoilovic, M. Forensic Sci. Int., 1991, 51, 289-96.

[3] Santucci, K.A.; Nelson, D.G.; McQuillen, K.K.; Duffy, S.J.; Linakis, J.G. Pediatrics, 1999, 104(6), 1342-4.

[4] Marshall, S.; Bennett, A.; Fraval, H. http://www.bvda.com/EN/ prdctinf/semenfluo.html (Sept 06, 2007).

[5] Wawryk, J.; Odell, M. J. Clin. Forensic Med., 2005, 12, 296-301.

[6] Vandenberg, N.; van Oorschot, R.A.H. J Forensic Sci., 2006, 51(2), 361-70.

[7] Soukos, N.S.; Crowley, K.; Bamberg, M.P.; Gilies, R.; Doukas, A.G.; Evans, R.; Kollias, N. Forensic Sci. Int., 2000, 114, 133-138.

[8] Auvdel, M.J. J. Forensic Sci.,1987, 32, 326-45.

[9] Kobus, H.J.; Silenieks, E.; Scharnberg, J. J. Forensic Sci., 2002, 47, 819-23.

[10] Schenck, M.; Kolbe, E. Grundriß der physiologischen Chemie; Gustav Fischer: Jena, 1990.

[11] Pschyrembel, W. Pschyrembel - Klinisches Wörterbuch, 259th ed.; Walter de Gryter: Berlin, 2002.

[12] Aps, J.K.M.; Martens, L.C. Forensic Sci. Int., 2005, 150, 119-131. 